\title{
Renal mucinous tubular and spindle cell carcinoma
}

\author{
Amel Trabelsi, MD; Wided Stita, MD; Mohamed Tahar Yacoubi, MD; Soumaya Rammeh, MD; \\ Sihem Hmissa, MD; Sadok Korbi, MD
}

\begin{abstract}
We report a case of mucinous tubular and spindle cell carcinoma in a 66-yearold woman. The tumour, located on the left kidney, was well circumscribed. Microscopically, the tumour was composed of cuboidal cells arranged in tubules and making abrupt transitions to spindle cell morphology in a myxoid stroma. Tumour cells were strongly positive for cytokeratin 7 and epithelial membrane antigen. Because of the favourable prognosis with this type of tumour, mucinous tubular and spindle cell carcinoma must be differentiated from papillary renal cell carcinoma, especially that with sarcomatoid change. No tumour recurrence or metastasis were reported with a follow-up of 23 months.
\end{abstract}

Can Urol Assoc J 2008;2(6):635-6

\section{Introduction}

Mucinous tubular and spindle cell carcinoma is a rare kidney tumour with a favourable prognosis. We report a new case in a 66-year-old woman.

\section{Case report}

A 66-year-old woman presented with a 1-month history of left flank pain. Renal function test results were normal. A computed tomography scan of the abdomen revealed a 6-cm well-circumscribed left renal mass contained in the renal capsule.

Left large nephrectomy (surgical excision of the kidney, the adrenal gland and the perirenal fat) was performed. Grossly, the kidney was occupied by a firm and well-circumscribed tumour that measured $6 \times 5 \times 5 \mathrm{~cm}$. The cut surface was tan-brown with foci of hemorrhage (Fig. 1). Microscopically, the tumour was composed of long cords and microtubules in a myxoid stroma (Fig. 2) with luminal mucin. Tumour cells were cuboidal, making abrupt transitions to spindle cell morphology (Fig. 3). Nuclear pleomorphism was absent in both cuboidal and spindle cells (Fig. 4). The myxoid stroma reacted strongly with alcian blue (Fig. 5).

Immunohistochemically, most of the tumour cells, including the spindle type, were strongly positive for cytokeratin 7 and epithelial membrane antigen. There was no invasion of the hilar fat and the diagnosis of mucinous tubular and spindle cell carcinoma was then made. No tumour recurrence or metastasis were reported with a follow-up of 23 months.

\section{Discussion}

Mucinous tubular and spindle cell carcinoma is a rare and only recently described tumour. Only 46 cases have been reported. ${ }^{1}$ This tumour is seen in adults, predominantly in women, and is typically detected as an asymptomatic renal mass. ${ }^{2}$

Grossly, mucinous tubular and spindle cell carcinoma is usually well circumscribed with a solid grey to white appearance to the cut surface. $^{2}$

Histologically, the tumour is composed of cuboidal cells arranged in microtubules and long cords making abrupt transitions to spindle morphology. ${ }^{2}$ These structures are arrayed in a mucinous or myxoid stroma that reacts strongly with alcian blue. ${ }^{2}$ Nuclear atypia and mitoses are rare in both cuboidal and spindle cells. Because of the presence of compact tubular architecture, focal papillations and mucin production, the mucinous tubular and spindle cell carcinoma has some morphological similarities with papillary renal cell carcinoma, particularly type 1,2 but extracellular mucin is rare or absent in the latter.

This tumour can also be confused with the papillary renal cell carcinoma that has sarcomatoid change, but in the mucinous tubular and spindle cell carcinoma, spindle cells are arranged in parallel bundles with eosinophilic cytoplasm and low-grade nuclei. ${ }^{3}$

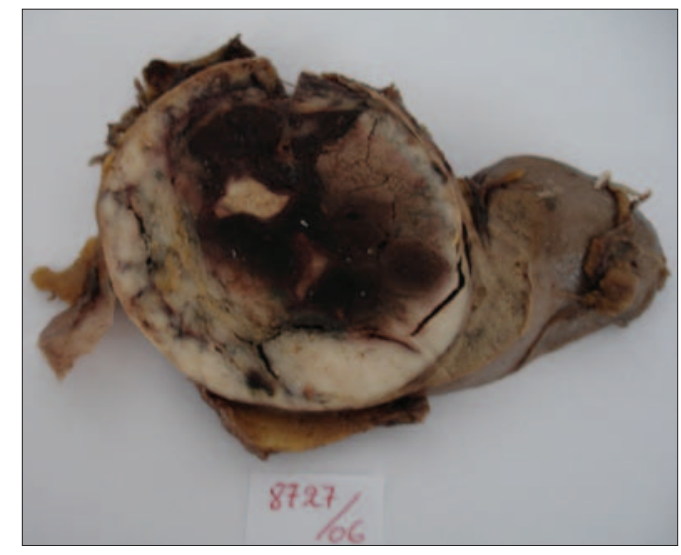

Fig. 1. The cut surface of the tumour was tan-brown with foci of hemorrhage. 
Trabelsi et al.

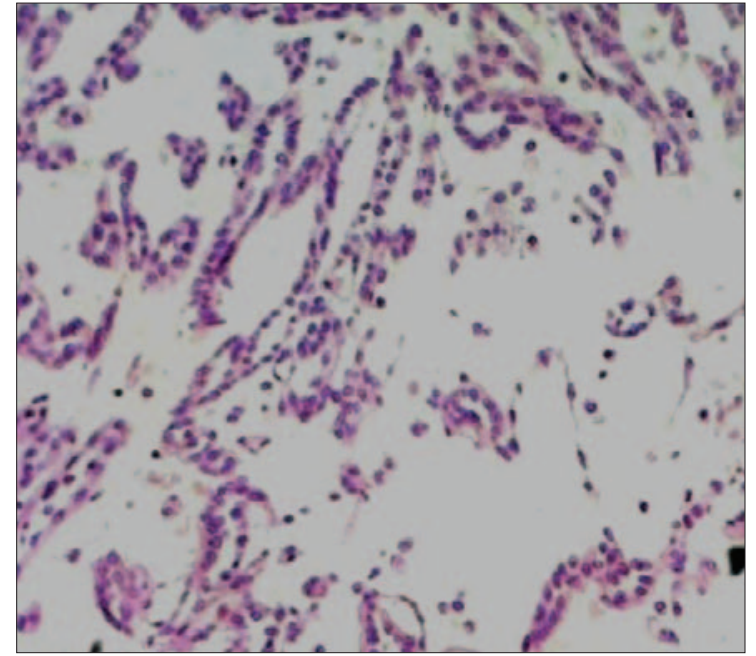

Fig. 2. Histological section showing long cords and microtubules in a myxoid stroma (hematoxylin-eosin stain, original magnification $\times 200$ ).

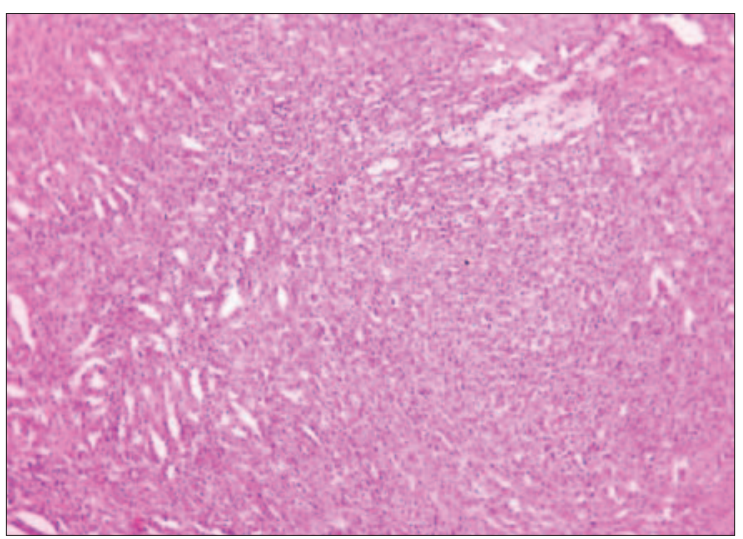

Fig. 3. Histological section showing cuboidal cells making abrupt transitions to spindle cell morphology (hematoxylin-eosin stain, original magnification $\times 100$ ).

Immunohistochemistry is not helpful in discriminating between papillary renal cell carcinoma and mucinous tubular and spindle cell carcinoma. The morphological interpretation is still important in the distinction between these tumours.

In summary, mucinous tubular and spindle cell carcinoma is a rare and only recently described distinctive subtype of renal cell carcinoma. It must be differentiated from papillary renal cell carcinoma, especially that with sarcomatoid change, which has a much poorer prognosis.

From the Department of Pathology, CHU Farhat Hached, Sousse, Tunisia This article has been peer reviewed.

Competing interests: None declared.

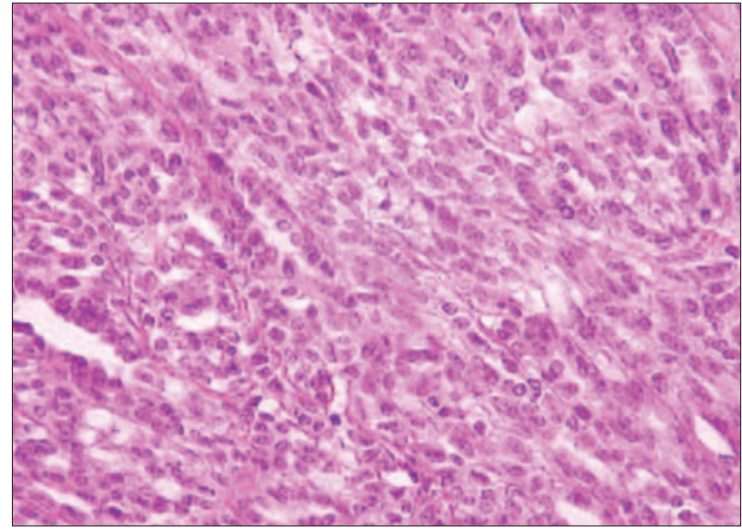

Fig. 4. Histological section showing absence of nuclear pleomorphism (hematoxylin-eosin stain, original magnification $\times 400$ ).

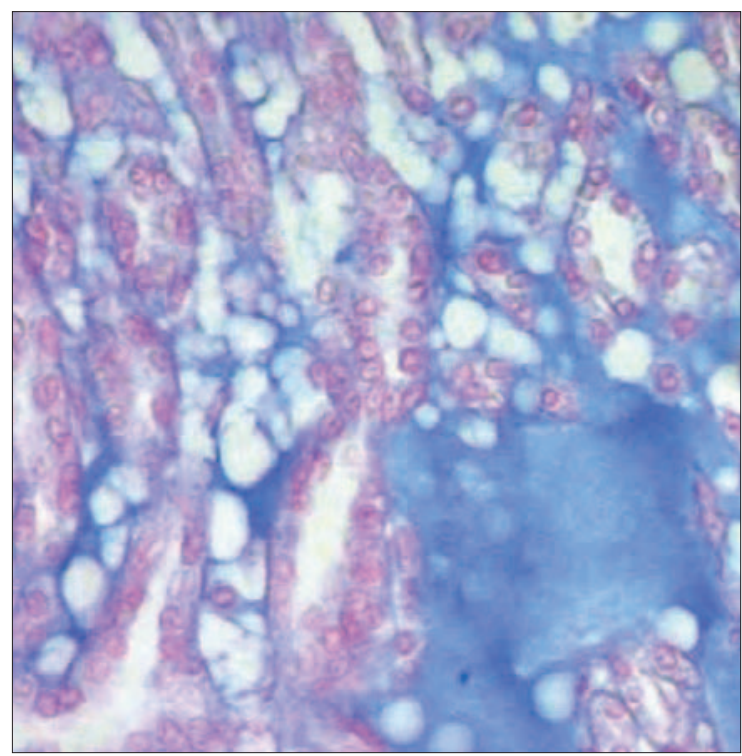

Fig. 5. Histological section showing myxoid stroma reaction with alcian blue (hematoxylin-eosin stain, original magnification $\times 400$ ).

\section{References}

1. Paner GP, Srigley JR, Radhakrishnan A, et al. Immunohistochemical analysis of mucinous tubular and spindle cell carcinoma and papillary renal cell carcinoma of the kidney, significant immunophenotypic overlap warrants diagnostic caution. Am I Surg Pathol 2006;30:13-9.

2. Eble JN. Mucinous tubular and spindle cell carcinoma and post-neuroblastoma carcinoma: newly recognised entities in the renal cell carcinoma family. Pathology 2003; 35:499-504.

3. Fleming S. Recently recognized epithelial tumours of the kidney. Curr Diagn Pathol 2005;11:162-9.

Correspondence: Dr. Amel Trabelsi, Department of Pathology, CHU Farhat Hached, Sousse, Tunisia; trabelsiamel@yahoo.fr 